\title{
Quantum entanglement between two magnon modes via Kerr nonlinearity driven far from equilibrium
}

\author{
Zhedong Zhang $\odot{ }^{1, *}$ Marlan O. Scully, ${ }^{1,2,3}$ and Girish S. Agarwal ${ }^{1, \dagger}$ \\ ${ }^{1}$ Texas A\&M University, College Station, Texas 77843, USA \\ ${ }^{2}$ Baylor University, Waco, Texas 76704, USA \\ ${ }^{3}$ Princeton University, Princeton, New Jersey 08544, USA
}

(Received 20 May 2019; revised manuscript received 21 July 2019; published 19 September 2019)

\begin{abstract}
We propose a scheme to entangle two magnon modes via the Kerr nonlinear effect when driving the systems far from equilibrium. We consider two macroscopic yttrium iron garnets interacting with a single-mode microcavity through the magnetic dipole coupling. The Kittel mode describing the collective excitations of a large number of spins is excited through the driving cavity with a strong microwave field. We demonstrate how Kerr nonlinearity creates the entangled quantum states between the two macroscopic ferromagnetic samples, when the microcavity is strongly driven by a blue-detuned microwave field. Such quantum entanglement survives at the steady state. Our work offers insights and guidance in designing experiments for observing entanglement in massive ferromagnetic materials. It can also find broad applications in macroscopic quantum effects and magnetic spintronics.
\end{abstract}

DOI: 10.1103/PhysRevResearch.1.023021

\section{INTRODUCTION}

Recent advances in ferromagnetic materials have drawn considerable attention in studies of quantum nature in magnetic systems, as the limitations of electrical circuitry are reached. Thanks to the low loss of the collective excitations of spins known as magnons in magnetic samples, the magnons offer a new paradigm for developing future generations of spintronic devices and quantum engineering [1-6]. Yttrium iron garnet (YIG) with a size of $\sim 100 \mu \mathrm{m}$ as fabricated in recent experiments provides new insights for studying macroscopic quantum effects, such as entanglement and squeezing, that have raised widespread interest in different branches of physics during the last decade [7-12]. Quantum entanglement between massive mirror and optical cavity photons has been explored, in both theoretical and experimental aspects [13-18]. Several following ideas have suggested the extension of such an entangled quantum state to magnons in the microwave regime, due to their great potential for macroscopic spintronic devices. Many experimental efforts have been devoted to the quantum nature of magnon states, through hybridizing the spin waves with other degrees of freedom, e.g., superconducting qubits and phonon modes [19-22]. Compared to atoms and photonics, magnonics holds the potential for implementing quantum states in more

\footnotetext{
*zhedong.zhang@tamu.edu

† girish.agarwal@tamu.edu
}

Published by the American Physical Society under the terms of the Creative Commons Attribution 4.0 International license. Further distribution of this work must maintain attribution to the author(s) and the published article's title, journal citation, and DOI. massive objects. This can be seen from the 320- $\mu \mathrm{m}$-diameter YIG spheres implemented in recent experiments [23].

Ferromagnetic materials provide a promising platform for studying the hybrid quantum systems in diversity, due to the rich interactions including magnetic dipole, magneto-optical, and magnetostrictive couplings that allow one to implement the interactions between drastically different physical systems [24]. Of particular interest are magnon polaritons, where strong and even ultrastrong light-matter couplings can be achieved, along with the fact of their high spin density and low dissipation rate [25-31]. This may serve as a potential candidate for implementing quantum information transducers and memories [31,32]. For the architecture of quantum magnonics, the macroscopic quantum effects are essentially worthy of being explored. The most recent work using drivendissipation theory suggests magnon-photon-phonon entanglement and also the squeezing of magnon modes in which both the entanglement and squeezing are essentially transferred into the mechanical mode [33-35]. From a theoretical point of view, this macroscopic quantum nature of magnon modes stems from the nonlinearity that can be enhanced by driving the systems far from equilibrium. Two prominent schemes are responsible for introducing such nonlinearity: the magnetostrictive interaction and the Kerr effect, where the latter results from the magnetocrystalline anisotropy. Apart from the magnon-phonon interaction, Kerr nonlinearity plays a significant role in magnon spintronics [5]. Recent experiments in YIG spheres demonstrated the multistability and photon-mediated control of spin current, due to the Kerr effect [36-38]. These studies are essentially semiclassical. In contrast, the current article is to show how Kerr nonlinearity can be used to produce remarkable quantum features of YIG bulks.

In this article, we propose a scheme of entangling magnon modes in two massive YIG spheres via Kerr nonlinearity. 
The two magnon modes interact with a microcavity through the beam-splitter-like coupling, which cannot produce any entanglement. Nevertheless, activating Kerr nonlinearity via strong driving results in squeezing-like coupling which may let the magnon become entangled with cavity photons. The subsequent entanglement transfer between photons and the other magnon mode will lead to the entanglement between magnon modes. The condition for optimizing the magnonmagnon entanglement is found and is confirmed by our numerical calculations. By taking into account the experimentally feasible parameters, we show that considerable magnonmagnon entanglement can be created. Such entanglement is also shown to be robust against cavity leakage. Our work offers insights and perspectives for studying the quantum effects in complex molecules. These have been manifested by the excited-state dynamics in dye molecules and even bacteria, implying the entangled quantum states when interacting with microcavities [39-43].

\section{MODEL AND EQUATION OF MOTION}

We consider a hybrid magnon-cavity system consisting of two bulk ferromagnetic materials and one microwave cavity mode (see Fig. 1). The ferromagnetic sample contains dispersive spin waves, in which only the spatially uniform mode (Kittel mode [44]) is assumed to strongly interact with cavity photons. This is reasonable as the magnetostatic mode (MS) with finite wave number has a distinct frequency from the Kittel mode so that the selective excitation may be implemented through the driving field wavelength and cavity mode selection [45]. Hence the full Hamiltonian of this cavity magnonics system reads

$$
\begin{aligned}
H= & -\int M_{z} B_{0} d \mathbf{r}-\frac{\mu_{0}}{2} \int M_{z} H_{\mathrm{an}} d \mathbf{r} \\
& +\frac{1}{2} \int\left(\varepsilon_{0} \mathbf{E}^{2}+\frac{\mathbf{B}^{2}}{\mu_{0}}\right) d \mathbf{r}-\int \mathbf{M} \cdot \mathbf{B} d \mathbf{r},
\end{aligned}
$$

where $\mathbf{B}_{0}=B_{0} \mathbf{e}_{z}$ is the applied static magnetic field and $\mathbf{M}=\gamma \hbar \mathbf{S} / V_{m}$ with $\gamma=e / m_{e}$ denoting the gyromagnetic ratio. $\mathbf{S}$ stands for the collective spin operator, spanning the

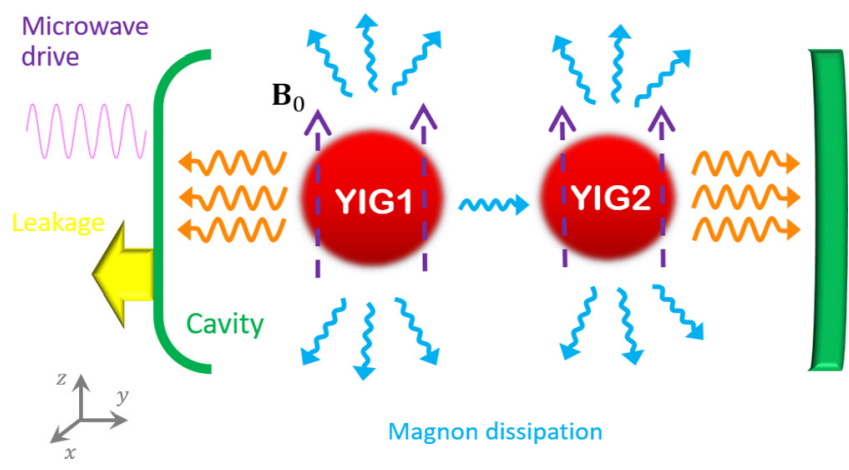

FIG. 1. Schematic of cavity magnons. Two YIG spheres are interacting with the basic mode of the microcavity in which the right mirror is made of high-reflection material so that photons leak from the left side. The static magnetic field for producing the Kittel mode is along the $z$ axis whereas the microwave driving and magnetic field inside the cavity are along the $x$ axis. $\operatorname{su}(2)$ Lie algebra $\left[S_{\alpha}, S_{\beta}\right]=i \varepsilon^{\alpha \beta \gamma} S_{\gamma} . V_{m}$ is the volume of ferromagnetic material. $\mathbf{B}$ represents the magnetic field inside the cavity. $\mathbf{H}_{\mathrm{an}}$ is the anisotropic field due to the magnetocrystalline anisotropy and has a $z$ component only owing to the crystallographic axis being aligned along the applied static magnetic field. Thereby the anisotropic field is given by $H_{\mathrm{an}}=-2 K_{\mathrm{an}} M_{z} / M^{2}$, where $K_{\mathrm{an}}$ and $M$ denote the dominant first anisotropy constant and the saturation magnetization, respectively. One can recast the Hamiltonian in Eq. (1) into

$$
\begin{aligned}
H= & -\gamma \hbar \sum_{j=1}^{2} B_{j, 0} S_{j, z}+\gamma^{2} \hbar^{2} \sum_{j=1}^{2} \frac{\mu_{0} K_{\mathrm{an}}^{(j)}}{M_{j}^{2} V_{j, m}} S_{j, z}^{2} \\
& +\hbar \omega_{c} a^{\dagger} a-\gamma \hbar \sum_{j=1}^{2} S_{j, x} B_{j, x}
\end{aligned}
$$

by assuming that the magnetic field inside the cavity is along the $x$ axis. The Holstein-Primakoff transform yields $S_{i, z}=S_{i}-m_{i}^{\dagger} m_{i}, \quad S_{i,+}=\left(2 S_{i}-m_{i}^{\dagger} m_{i}\right)^{1 / 2} m_{i}, \quad S_{i,-}=$ $m_{i}^{\dagger}\left(2 S_{i}-m_{i}^{\dagger} m_{i}\right)^{1 / 2}$, where $S_{i, \pm} \equiv S_{i, x} \pm i S_{i, y}$ and $m_{i}$ represents the bosonic annihilation operator [46]. For the yttrium iron garnets (YIGs) with diameter $d=40 \mu \mathrm{m}$, the density of the ferric ion $\mathrm{Fe}^{3+}$ is $\rho=4.22 \times 10^{27} \mathrm{~m}^{-3}$, which leads to the total spin $S=\frac{5}{2} \rho V_{m}=7.07 \times 10^{14}$. This is often much larger than the number of magnons, so that we can safely approximate $S_{j,+} \simeq \sqrt{2 S_{j}} m_{j}, S_{j,-} \simeq \sqrt{2 S_{j}} m_{j}^{\dagger}$. In the presence of the external microwave driving field, the effective Hamiltonian of the hybrid magnon-cavity system is of the form [47]

$$
\begin{aligned}
H_{\mathrm{eff}}= & \hbar \omega_{c} a^{\dagger} a+\hbar \sum_{j=1}^{2}\left[\omega_{j} m_{j}^{\dagger} m_{j}+g_{j}\left(m_{j}^{\dagger} a+m_{j} a^{\dagger}\right)\right. \\
& \left.+\Delta_{j} m_{j}^{\dagger} m_{j} m_{j}^{\dagger} m_{j}\right]+i \hbar \Omega\left(a^{\dagger} e^{-i \omega_{d} t}-a e^{i \omega_{d} t}\right),
\end{aligned}
$$

where $\delta_{s}=\omega_{s}-\omega_{d}, \quad \delta_{c}=\omega_{c}-\omega_{d}$, and the cavity frequency is denoted by $\omega_{c}$. The frequency of the Kittel mode is $\omega_{s}=\gamma B_{s, 0}-2 \hbar \mu_{0} K_{\text {an }}^{(s)} \gamma^{2} S_{s} / M_{s}^{2} V_{s, m}$ with $\gamma / 2 \pi=$ $28 \mathrm{GHz} / \mathrm{T}$. $g_{s}$ gives the magnon-cavity coupling and $\Delta_{s}=$ $\mu_{0} K_{\mathrm{an}}^{(s)} \gamma^{2} / M_{s}^{2} V_{s, m}$ gives the Kerr nonlinearity. The Rabi frequency $\Omega=\sqrt{2 P_{d} \gamma_{c} / \hbar \omega_{d}}$ in the last term quantifies the strength of the field inside microcavity driven by the microwave magnetic field, where $P_{d}$ and $\omega_{d}$ represent the power and frequency of the microwave field, respectively. The quantum Langevin equations (QLEs) for the hybrid cavity-magnon system are given by

$$
\begin{gathered}
\dot{m}_{s}=-\left(i \delta_{s}+\gamma_{s}\right) m_{s}-2 i \Delta_{s} m_{s}^{\dagger} m_{s} m_{s}-i g_{s} a+\sqrt{2 \gamma_{s}} m_{s}^{\text {in }}(t), \\
\dot{a}=-\left(i \delta_{c}+\gamma_{c}\right) a-i \sum_{j=1}^{2} g_{j} m_{j}+\Omega+\sqrt{2 \gamma_{c}} a^{\text {in }}(t),
\end{gathered}
$$

where $\gamma_{c}$ and $\gamma_{s}$ represent the rates of cavity leakage and magnon dissipation, respectively. $m_{s}^{\text {in }}(t)$ and $a^{\text {in }}(t)$ are the input noise operators having zero mean and white noise: $\left\langle m_{s}^{\text {in, }} \dagger(t) m_{s}^{\text {in }}\left(t^{\prime}\right)\right\rangle=\bar{n}_{s} \delta\left(t-t^{\prime}\right), \quad\left\langle m_{s}^{\text {in }}(t) m_{s}^{\text {in }}, \dagger\left(t^{\prime}\right)\right\rangle=\left(\bar{n}_{s}+\right.$ 1) $\delta\left(t-t^{\prime}\right) ; \quad\left\langle a^{\text {in }, \dagger}(t) a^{\text {in }}\left(t^{\prime}\right)\right\rangle=0,\left\langle a^{\text {in }}(t) a^{\text {in }, \dagger}\left(t^{\prime}\right)\right\rangle=\delta\left(t-t^{\prime}\right)$, where $\bar{n}_{s}=\left[\exp \left(\hbar \omega_{s} / k_{B} T\right)-1\right]^{-1}$ denotes the Planck factor of the $s$ th magnon mode.

Since the microcavity is under strong driving by the microwave field, the beam-splitter-like coupling between 
magnons and cavity leads to the large amplitudes of both magnon and cavity modes, namely, $\left|\left\langle m_{s}\right\rangle\right|,|\langle a\rangle| \gg 1$. In this case, one can safely introduce the expansion $m_{s}=\left\langle m_{s}\right\rangle+$ $\delta m_{s}, \quad a=\langle a\rangle+\delta a$ in the vicinity of the steady state, by neglecting the higher-order fluctuations of the operators. We thereby obtain the linearized QLEs for the quadratures $\delta X_{s}, \delta Y_{s}, \delta X, \delta Y$ defined as $\delta X_{1}=\left(\delta m_{1}+\delta m_{1}^{\dagger}\right) / \sqrt{2}, \delta Y_{1}=$ $\left(\delta m_{1}-\delta m_{1}^{\dagger}\right) / i \sqrt{2}, \quad \delta X_{2}=\left(\delta m_{2}+\delta m_{2}^{\dagger}\right) / \sqrt{2}, \quad \delta Y_{2}=\left(\delta m_{2}-\right.$

$$
\begin{gathered}
\left.\delta m_{2}^{\dagger}\right) / i \sqrt{2}, \delta X=\left(\delta a+\delta a^{\dagger}\right) / \sqrt{2}, \delta Y=\left(\delta a-\delta a^{\dagger}\right) / i \sqrt{2}: \\
\dot{\sigma}(t)=A \sigma(t)+f(t),
\end{gathered}
$$

where $\sigma(t)=\left[\delta X_{1}(t), \delta Y_{1}(t), \delta X_{2}(t), \delta Y_{2}(t), \delta X(t), \delta Y(t)\right]^{\mathrm{T}}$ and $f(t)=\left[\sqrt{2 \gamma_{1}} X_{1}^{\text {in }}(t), \sqrt{2 \gamma_{1}} Y_{1}^{\text {in }}(t), \sqrt{2 \gamma_{2}} X_{2}^{\text {in }}(t), \sqrt{2 \gamma_{2}} Y_{2}^{\text {in }}(t)\right.$, $\left.\sqrt{2 \gamma_{c}} X^{\text {in }}(t), \sqrt{2 \gamma_{1}} Y^{\text {in }}(t)\right]^{\mathrm{T}}$ are the vectors for quantum fluctuations and noise, respectively. The drift matrix reads

$$
A=\left(\begin{array}{cccccc}
F_{1}-\gamma_{1} & \tilde{\delta}_{1}-G_{1} & 0 & 0 & 0 & g_{1} \\
-\tilde{\delta}_{1}-G_{1} & -F_{1}-\gamma_{1} & 0 & 0 & -g_{1} & 0 \\
0 & 0 & F_{2}-\gamma_{2} & \tilde{\delta}_{2}-G_{2} & 0 & g_{2} \\
0 & 0 & -\tilde{\delta}_{2}-G_{2} & -F_{2}-\gamma_{2} & -g_{2} & 0 \\
0 & g_{1} & 0 & g_{2} & -\gamma_{c} & \delta_{c} \\
-g_{1} & 0 & -g_{2} & 0 & -\delta_{c} & -\gamma_{c}
\end{array}\right)
$$

with the magnetocrystalline anisotropy quantified by $G_{s}=$ $2 \Delta_{s} \operatorname{Re}\left\langle m_{s}\right\rangle^{2}, F_{s}=2 \Delta_{s} \operatorname{Im}\left\langle m_{s}\right\rangle^{2}$, and the effective detuning of magnons $\tilde{\delta}_{s}=\delta_{s}+2 \sqrt{G_{s}^{2}+F_{s}^{2}}=\delta_{s}+4 \Delta_{s}\left|\left\langle m_{s}\right\rangle\right|^{2}$, which includes the frequency shift caused by Kerr nonlinearity. The means $\left\langle m_{1,2}\right\rangle$ are given by

$$
\left\langle m_{1}\right\rangle=\frac{i g_{1} \Omega}{\left(\tilde{\delta}_{1}-i \gamma_{1}\right)\left(\delta_{c}-i \gamma_{c}\right)-g_{1}^{2}-\frac{g_{2}^{2}\left(\tilde{\delta}_{1}-i \gamma_{1}\right)}{\delta_{2}-i \gamma_{2}}},
$$

and $(1 \leftrightarrow 2)$.

Before studying entanglement, it is essential to elucidate the mechanism for optimizing the entanglement via Kerr nonlinearity. To this end, we proceed via the effective Hamiltonian for quantum fluctuations

$$
\begin{aligned}
H_{\mathrm{qf}}= & \hbar \sum_{s=1}^{2}\left[\tilde{\delta}_{s} \delta m_{s}^{\dagger} \delta m_{s}+\tilde{\Delta}_{s} \delta m_{s}^{\dagger} \delta m_{s}^{\dagger}+\tilde{\Delta}_{s}^{*} \delta m_{s} \delta m_{s}\right. \\
& \left.+g_{s}\left(\delta m_{s}^{\dagger} \delta a+\delta m_{s} \delta a^{\dagger}\right)\right]+\hbar \delta_{c} \delta a^{\dagger} \delta a,
\end{aligned}
$$

where $\tilde{\Delta}_{s}=\left(G_{s}+i F_{s}\right) / 2$. The quadratic terms $\delta m_{s}^{\dagger} \delta m_{s}^{\dagger}, \quad \delta m_{s} \delta m_{s}$ imply the effective magnon-magnon interaction induced by the magnetocrystalline anisotropy, which may be significantly enhanced by strong driving. This, in fact, is responsible for the entanglement. To make it elaborate, let us introduce the Bogoliubov transformation $[48,49] \quad \delta \beta_{s}=u_{s} \delta m_{s}-v_{s}^{*} \delta m_{s}^{\dagger}, \quad \delta \beta_{s}^{\dagger}=-v_{s} \delta m_{s}+u_{s}^{*} \delta m_{s}^{\dagger}$, where $\quad u_{s}=\sqrt{\frac{1}{2}\left(\frac{\delta_{s}}{\varepsilon_{s}}+1\right)}, \quad v_{s} e^{i \alpha}=-\sqrt{\frac{1}{2}\left(\frac{\delta_{s}}{\varepsilon_{s}}-1\right)}, \quad \alpha=$ $\arctan \left(F_{s} / G_{s}\right)$, and $\varepsilon_{s}=\left(\tilde{\delta}_{s}^{2}-4\left|\tilde{\Delta}_{s}\right|^{2}\right)^{1 / 2}$. Inserting these into Eq. (8) we find

$$
\begin{aligned}
H_{\mathrm{qf}}= & \hbar \sum_{s=1}^{2}\left[\varepsilon_{s} \delta \beta_{s}^{\dagger} \delta \beta_{s}+g_{s}\left(\left(v_{s} \delta \beta_{s}+u_{s} \delta \beta_{s}^{\dagger}\right) \delta a\right.\right. \\
& \left.\left.+\left(u_{s}^{*} \delta \beta_{s}+v_{s}^{*} \delta \beta_{s}^{\dagger}\right) \delta a^{\dagger}\right)\right]+\hbar \delta_{c} \delta a^{\dagger} \delta a,
\end{aligned}
$$

which shows that $\varepsilon_{s} \simeq-\delta_{c}$ is optimal for the entanglement, due to the magnon-photon squeezing term $g_{s}\left(v_{s} \delta \beta_{s} \delta a+\right.$ $\left.v_{s}^{*} \delta \beta_{s}^{\dagger} \delta a^{\dagger}\right)$. This will be confirmed by the later numerical results when taking into account experimental parameters.

\section{ENTANGLEMENT BETWEEN MAGNON MODES}

Since we are using the linearized quantum Langevin equations, the Gaussian nature of the input states will be preserved during the time evolution of systems. The quantum fluctuations are thus the continuous three-mode Gaussian state, which is completely characterized by a $6 \times 6$ covariance matrix $(\mathrm{CM})$ defined as $C_{i j}\left(t, t^{\prime}\right)=\frac{1}{2}\left\langle\sigma_{i}(t) \sigma_{j}\left(t^{\prime}\right)+\sigma_{j}\left(t^{\prime}\right) \sigma_{i}(t)\right\rangle$, $(i, j=1,2, \ldots, 6)$, where the average is taken over the system and bath degrees of freedoms. Suppose the drift matrix $A$ is negatively defined; the solution to Eq. (5) is $\sigma(t)=$ $M(t) \sigma(0)+\int_{0}^{t} M(s) f(t-s) d s$, where $M(t)=\exp (A t)$. This enables us to find the equation which the CM obeys,

$$
\dot{C}(t+\tau, t)=A C(t+\tau, t)+C(t+\tau, t) A^{\mathrm{T}}+e^{A \tau} D,
$$

for $\tau \geqslant 0$. Thus the stationary CM can be straightforwardly obtained by letting $\tau=0, t \rightarrow \infty$ in Eq. (10) that yields the Lyapunov equation

$$
A C_{\infty}+C_{\infty} A^{\mathrm{T}}=-D
$$

where the diffusion matrix is $D=\operatorname{diag}\left[\gamma_{1}\left(2 \bar{n}_{1}+1\right), \gamma_{1}\left(2 \bar{n}_{1}+\right.\right.$ 1), $\left.\gamma_{2}\left(2 \bar{n}_{2}+1\right), \gamma_{2}\left(2 \bar{n}_{2}+1\right), \gamma_{c}, \gamma_{c}\right]$ defined through $\left\langle f_{i}(t) f_{j}\left(t^{\prime}\right)+f_{j}\left(t^{\prime}\right) f_{i}(t)\right\rangle=2 D_{i j} \delta\left(t-t^{\prime}\right)$.

\section{A. Magnon-magnon and magnon-photon entanglements}

To study the bipartite magnon-magnon and magnonphoton entanglements, we adopt the logarithmic negativity $E_{N}$ by computing the $4 \times 4 \mathrm{CM}$ related to the two modes of interest. This can be achieved by defining $E_{N}=\max \left[0,-\ln 2 v_{-}\right]$, where $v_{-}=\min \mid$ eig $\oplus_{j=1}^{2}\left(-\sigma_{y}\right) P_{12} C_{4} P_{12} \mid$ and $\sigma_{y}$ is the Pauli matrix [50,51]. $C_{4}$ is the $\mathrm{CM}$ of two subsystems, obtained through removing in $C_{\infty}$ solved from Eq. (11) the rows and columns of the uninteresting modes. The matrix $P_{12}=\sigma_{z} \oplus 1$ realizes the partial transposition at the level of the $\mathrm{CM}$. In what follows, we will work in the monostable scheme of magnons. Furthermore, we will focus on the case of two identical magnons having $G_{1,2}=G, F_{1,2}=F, \tilde{\delta}_{1,2}=\tilde{\delta}, \Delta_{1,2}=$ $\Delta, g_{1,2}=g$. 
(a)

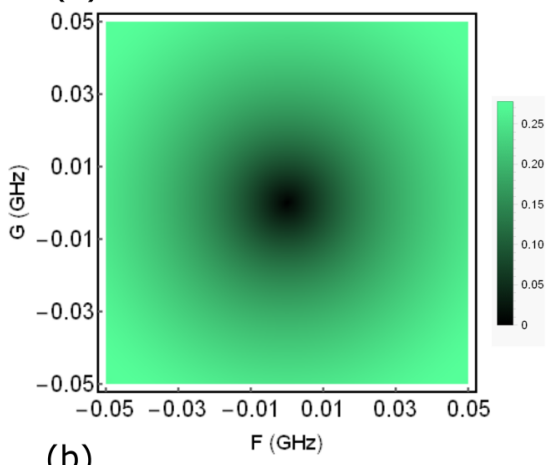

(b)

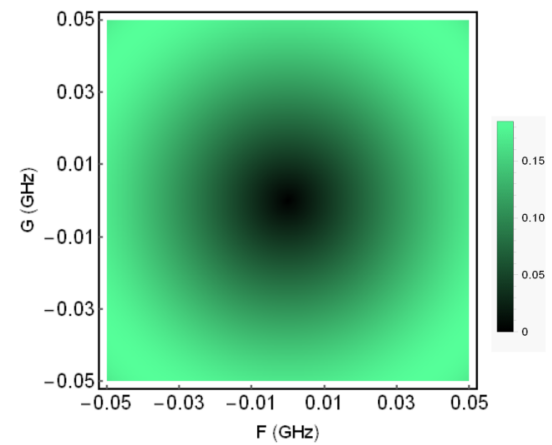

(c)

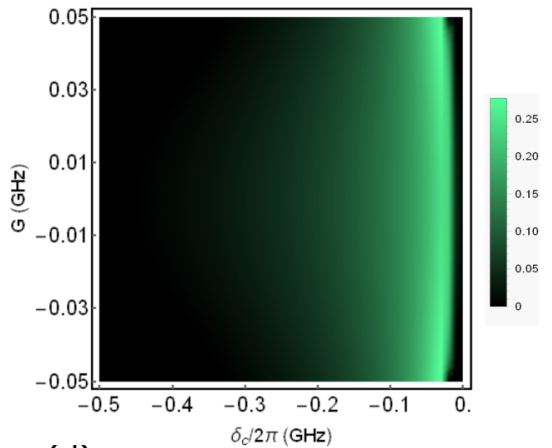

(d)

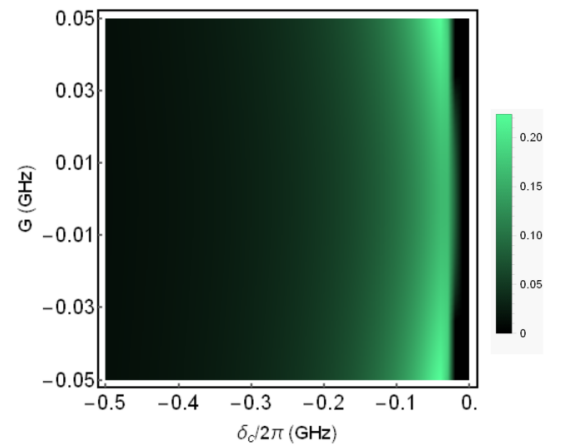

(e)

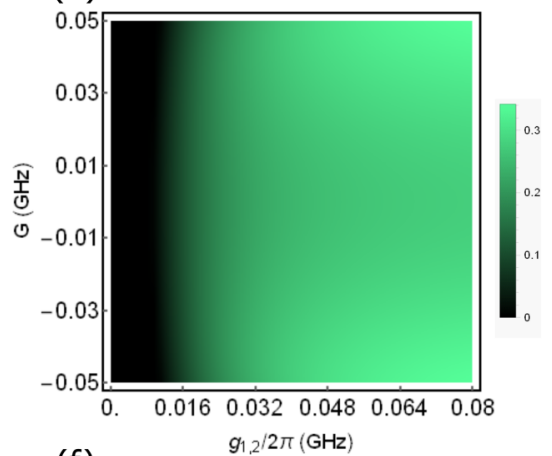

(f)

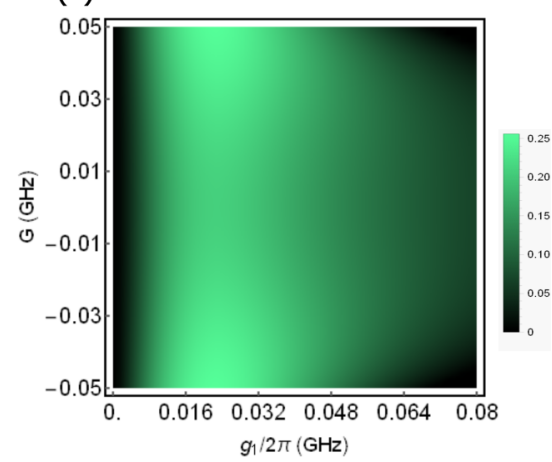

FIG. 2. Two-dimensional plots for (top) magnon-magnon entanglement $E_{m_{1} m_{2}}$ and (bottom) magnon-cavity entanglement $E_{m_{1} a}$ when turning off the coupling between the cavity and the second sphere $\left(g_{2}=0\right)$. (a) $g_{1,2} / 2 \pi=41 \mathrm{MHz}, \delta_{c} / 2 \pi=-0.03 \mathrm{GHz}$; (b) $g_{1} / 2 \pi=$ $41 \mathrm{MHz}, g_{2}=0, \delta_{c} / 2 \pi=-0.03 \mathrm{GHz}$; (c) $F_{1,2}=-0.048 \mathrm{GHz}, g_{1,2} / 2 \pi=41 \mathrm{MHz}$; (d) $F_{1,2}=-0.048 \mathrm{GHz}, g_{1} / 2 \pi=41 \mathrm{MHz}, g_{2}=0$; and (e), (f) $F_{1,2}=-0.048 \mathrm{GHz}, \delta_{c} / 2 \pi=-0.03 \mathrm{GHz}$. Other parameters are $\omega_{1,2} / 2 \pi=10 \mathrm{GHz}, \delta_{1,2} / 2 \pi=-1 \mathrm{MHz}, \gamma_{1,2} / 2 \pi=8.8 \mathrm{MHz}$, $\gamma_{c} / 2 \pi=1.9 \mathrm{MHz}$, and $T=10 \mathrm{mK}$.

Figure 2 shows the magnon-magnon entanglement versus some key parameters of the system. Here we have taken into account the experimentally feasible parameters [36] $\omega_{1,2} / 2 \pi=10 \mathrm{GHz}, \delta_{1,2} / 2 \pi=-1 \mathrm{MHz}, \gamma_{1,2} / 2 \pi=$ $8.8 \mathrm{MHz}$, and $\gamma_{c} / 2 \pi=1.9 \mathrm{MHz}$ for the YIG bulk at low temperature $T=10 \mathrm{mK}$. First of all we observe from Figs. 2(a) and 2(b) that Kerr nonlinearity is responsible for creating the steady-state entanglement between two magnon modes, evident from the fact that the entanglement dies out when $G=$ $F=0$. This results from the dominated beam-splitter interaction between magnon mode and cavity photons, once $G=$ $F=0$. Thereby no magnon-cavity entanglement can be created, as seen in Fig. 2(b). We take the condition $\varepsilon_{s} \simeq-\delta_{c}$ for optimizing the magnon-photon entanglement, as illustrated in Fig. 2(d), where $\varepsilon_{s} \simeq \sqrt{3\left(G_{s}^{2}+F_{s}^{2}\right)}$. The two-mode squeezing term $g_{s}\left(v_{s} \delta \beta_{s} \delta a+v_{s}^{*} \delta \beta_{s}^{\dagger} \delta a^{\dagger}\right)$ squeezes the joint state between one magnon mode and cavity photons, which results in the partial entanglement in between. Because the same type of interaction occurs when coupling the other magnon mode with the cavity, the two distanced magnon modes are expected to be entangled. This is confirmed in Fig. 2(c), manifesting the optimal magnon-magnon entanglement in the vicinity of $\varepsilon_{s} \simeq-\delta_{c}$. The elaborate transfer from magnon-photon entanglement to magnon-magnon entanglement is subsequently evident as the coupling of the cavity to another sphere is turned on. Since the biparticle entanglement originates from the Kerr nonlinearity quantified by $G_{1,2}$ and $F_{1,2}$, there must be an interplay between the couplings $G_{s}, F_{s}$, and $g_{s}$, which is depicted in Figs. 2(e) and 2(f). In Fig. 2(c) we take $g_{1,2} / 2 \pi=41 \mathrm{MHz}$ and this implies $\delta_{c} / 2 \pi \simeq-0.03 \mathrm{GHz}$ for the optimal entanglement $E_{m_{1} m_{2}}$. We then adopt the magnitude of $\delta_{c}$ for plotting Figs. 2(a) and 2(b). Using $\sqrt{G^{2}+F^{2}}=$ $2 \Delta|\langle m\rangle|^{2}$ and Eq. (7) for the 40- $\mu$ m-diameter YIG spheres, the optimal entanglement with $|G|=0.038 \mathrm{GHz}, \quad|F|=$ $0.028 \mathrm{GHz}$ [see Fig. 2(a)] yields the Rabi frequency $\Omega=$ $1.06 \times 10^{15} \mathrm{~Hz}$, corresponding to the drive power $P_{d}=314$ $\mathrm{mW}$. Indeed, the stronger nonlinearity will create more entanglement between the magnon modes. But we have to ensure the negatively defined matrix $A$ given in Eq. (6), and the condition $\left\langle m_{j}^{\dagger} m_{j}\right\rangle \ll 2 N_{j} s=5 \rho_{j} V_{j, m}$. It is easy to show how our procedure is valid under the parameter regimes we considered. Also, the experimental feasibility of an ultrastrong drive using microwave field needs consideration.

Figures 3(a) and 3(b) illustrate the entanglement between two magnon modes versus some controllable parameters by considering the $40-\mu \mathrm{m}$-diameter YIG sphere experiment, where $\omega_{1,2} / 2 \pi=10 \mathrm{GHz}, \delta_{1,2} / 2 \pi=-1 \mathrm{MHz}$, $\Delta_{1,2} / 2 \pi=1 \mu \mathrm{Hz}, g_{1,2} / 2 \pi=41 \mathrm{MHz}, \gamma_{1,2} / 2 \pi=8.8 \mathrm{MHz}$, and $\gamma_{c} / 2 \pi=1.9 \mathrm{MHz}$ have been taken according to Ref. [36]. We observe in Fig. 3(a) that for fixed driving power, the magnon-magnon entanglement is quite sensitive to cavity detuning $\delta_{c} \equiv \omega_{c}-\omega_{d}$, reaching its maximum at $\delta_{c} / 2 \pi \simeq$ $-0.03 \mathrm{GHz}$. This is consistent with the condition $\varepsilon_{j} \simeq-\delta_{c}$ as clarified for optimizing the entanglement. Figure 3(b) shows considerable entanglement when the system is driven far from equilibrium. This is reasonable because the strong external driving significantly enhances the Kerr nonlinearity that is responsible for both magnon-cavity squeezing and 

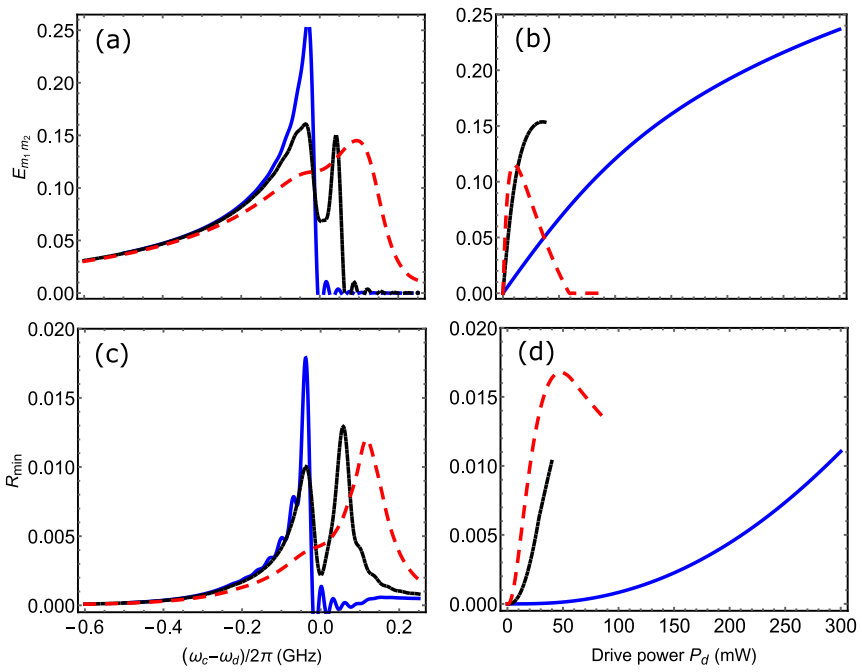

FIG. 3. Magnon-magnon and magnon-magnon-photon entanglements vary with (a), (c) cavity detuning and (b), (d) driving power. (a)-(d) Solid blue, dot-dashed black, and dashed red lines are for the cavity leakage $\gamma_{c} / 2 \pi=1.9 \mathrm{MHz}, 20 \mathrm{MHz}$, and $70 \mathrm{MHz}$, respectively; $g_{1,2} / 2 \pi=41 \mathrm{MHz}$. (a), (c) Solid blue, dot-dashed black, and dashed red lines also correspond to driving power $P_{d}=393 \mathrm{~mW}$, $38 \mathrm{~mW}$, and $11 \mathrm{~mW}$, respectively; (b), (d) $\delta_{c} / 2 \pi=-30 \mathrm{MHz}$. Other parameters are the same as Fig. 2.

entanglement, as elucidated in Eqs. (7) and (8). Notice the cutoff in both Figs. 3(b) and 3(d). This results from the requirement $\left\langle m_{j}^{\dagger} m_{j}\right\rangle \ll 2 N_{j} s=5 \rho_{j} V_{j, m}$ that ensures the validity of the magnon description for magnetic materials. Furthermore, Figs. 3(a) and 3(b) show that the weaker magnon-magnon entanglement is observed when increasing the cavity leakage. By noting the magnitude, we can still obtain some entanglement, even with a low-quality cavity showing weak magnoncavity coupling where $\gamma_{c}=8 \gamma_{1,2}>g_{1,2}$ is denoted by red dashed lines. This regime is crucial for detecting the entanglement used in Refs. [17,18], in which an additional cavity has a beam-splitter-like interaction with the magnon mode for reading out the magnon states associated with the CM. The transferred entanglement can then be measured through the homodyne detection by sending a weak microwave probe. This approach requires much larger cavity leakage than the magnon dissipation, namely, $\gamma_{c} \gg \gamma_{1,2}$, so that the magnon states can remain almost unchanged when switching off the laser driving.

\section{B. Magnon-magnon-photon entanglement}

We further study the tripartite magnon-magnon-photon entanglement quantified by minimal residual contangle defined as $[52,53]$

$$
\mathcal{R}_{i \mid j k}=C_{i \mid j k}-C_{i \mid j}-C_{i \mid k},
$$

where $C_{u \mid v}$ is the cotangle of subsystems $u$ and $v$ ( $v$ may involve one or two modes). This is a proper entanglement monotone defined as the squared logarithmic negativity, namely, $C_{u \mid v}=E_{u \mid v}^{2}$. The one-mode-versus-two-modes logarithmic negativity $E_{i \mid j k}$ is defined as

$$
E_{i \mid j k}=\max \left[0,-\ln 2 v_{i \mid j k}\right]
$$

along the line with the logarithmic negativity for bipartite entanglement. $v_{i \mid j k}=\min \left|\operatorname{eig} i \Omega_{3} \tilde{C}\right|$ is the minimum symplectic eigenvalue of the $6 \times 6 \mathrm{CM} \tilde{C}=P_{i \mid j k} C P_{i \mid j k}$ with the symplectic matrix $\Omega_{3}=\oplus_{j=1}^{3} i \sigma_{y} . C$ is the $6 \times 6 \mathrm{CM}$ of the full system. $P_{1 \mid 23}=\sigma_{z} \oplus 1 \oplus 1, P_{2 \mid 13}=1 \oplus \sigma_{z} \oplus 1$, and $P_{3 \mid 12}=1 \oplus 1 \oplus$ $\sigma_{z}$ are the matrices for partial transposition at the level of $6 \times 6 \mathrm{CM}$. The residue contangle satisfies the monogamy of quantum entanglement, that is, $\mathcal{R}_{i \mid j k} \geqslant 0$ and $C_{i \mid j k} \geqslant C_{i \mid j}+$ $C_{i \mid k}$, which is reminiscent of the Coffman-Kundu-Wootters monogamy inequality that holds for a system consisting of three qubits. A bona fide quantification of tripartite entanglement for Gaussian states is given by the minimum residual cotangle

$$
\mathcal{R}_{\min }=\min \left[\mathcal{R}_{m_{1} \mid m_{2} a}, \mathcal{R}_{m_{2} \mid m_{1} a}, \mathcal{R}_{a \mid m_{1} m_{2}}\right] \text {, }
$$

which guarantees the invariance of tripartite entanglement under all permutations of the modes.

In Figs. 3(c) and 3(d), one can observe the important role that Kerr nonlinearity plays in tripartite entanglement, besides the bipartite entanglement discussed above. Figure 3(c) shows that the tripartite entanglement is quite sensitive to cavity detuning $\delta_{c}=\omega_{c}-\omega_{d}$, reaching its maximum in the vicinity of $\delta_{c} / 2 \pi \simeq-0.03 \mathrm{GHz}$. This is consistent with the condition $\varepsilon_{j} \simeq-\delta_{c}$ as clarified for optimizing the entanglement in Eq. (9). Figure 3(d) shows the considerable tripartite entanglement when the system is driven far from equilibrium. This is attributed to the significant enhancement of Kerr nonlinearity by strong driving that is responsible for the magnon-cavity squeezing and entanglement. Furthermore, under the same parameter regime as in magnon-magnon entanglement, the magnon-magnon-photon entanglement is also quite robust against the cavity leakage.

The time-resolved detection of the photons emitting off the cavity axis may offer an alternative scheme for entanglement measurement. This leakage to the side is denoted by the blue wavy lines in Fig. 1. These photons arise from the decay of YIG's excitations quantified by $\gamma_{s}, s=1,2$, in Eq. (4). The quadrature information of magnon modes can be transferred to the time-gated emitted photons, which can be homodynely detected by interfering with an extra microwave field. This quantum-light-probe scheme may take advantage of being a noninvasive detection for entanglement measurement.

\section{CONCLUSION AND REMARKS}

In conclusion, we have proposed a protocol for entangling the magnon modes in two massive YIG spheres, through the Kerr nonlinearity that originates from the magnetocrystalline anisotropy. We show that such nonlinearity has to be essentially included to produce the entanglement. Our work demonstrates the stationary entanglement between two macroscopic YIG spheres driven far from equilibrium, within the experimentally feasible parameter regime. The amount of entanglement is quantified by the logarithmic negativity and is surprisingly robust against the cavity leakage: the entangled quantum state may persist with a low-quality cavity 
giving weak magnon-cavity coupling. This may be helpful to experimental design for entanglement measurement.

We should note that our idea for entangling magnon modes may be potentially extended to other complex systems, such as molecular aggregates and clusters, along with the fact of similar forms of nonlinear couplings $b^{\dagger} b q$ and $\Delta b^{\dagger} b b^{\dagger} b$. With the scaled-up parameters, the long-range entanglement in molecular aggregates would be anticipated, in that the exciton-exciton interaction is several orders of magnitude higher than the Kerr nonlinearity resulting from the magnetocrystalline anisotropy. For instance, the two-exciton coupling in J-aggregates and light-harvesting antennas takes the value of $\sim 50 \mathrm{~cm}^{-1}$, which is $\sim 0.3 \%$ of the exciton frequency. This is a much stronger nonlinearity than that in YIGs with Kerr coefficient $K \sim 0.1 \mathrm{nHz}$, that is, $\sim 10^{-11}$ of its Kittel frequency. Recent developments in both ultrafast spectroscopy and synthesis have revealed the important role of quantum coherence which may significantly modify the functions of complex molecules and may help the design of polaritonic molecular devices as well as polariton chemistry. Hence entangling the molecular aggregates may help studies of quantum phenomena in complex molecules.

\section{ACKNOWLEDGMENTS}

We gratefully acknowledge the support of AFOSR Award No. FA-9550-18-1-0141, ONR Award No. N00014-16-13054, and the Robert A. Welch Foundation (Awards No. A-1261 and No. A-1943-20180324). We also thank Jie Li and Tao Peng for useful discussions.
[1] Y. Kajiwara et al., Nature (London) 464, 262 (2010).

[2] L. J. Cornelissen, J. Liu, R. A. Duine, J. B. Youssef, and B. J. van Wees, Nat. Phys. 11, 1022 (2015).

[3] N. Zhu et al., Appl. Phys. Lett. 109, 082402 (2016).

[4] T. An et al., Nat. Mater. 12, 549 (2013).

[5] A. V. Chumak, V. I. Vasyuchka, A. A. Serga, and B. Hillebrands, Nat. Phys. 11, 453 (2015).

[6] A. V. Chumak, A. A. Serga, and B. Hillebrands, Nat. Commun. 5, 4700 (2014).

[7] M. Collet et al., Nat. Commun. 7, 10377 (2016).

[8] M. Ho, E. Oudot, J.-D. Bancal, and N. Sangouard, Phys. Rev. Lett. 121, 023602 (2018).

[9] R. Riedinger et al., Nature (London) 556, 473 (2018).

[10] H. Y. Yuan and M.-H. Yung, Phys. Rev. B 97, 060405(R) (2018)

[11] T. Morimae, A. Sugita, and A. Shimizu, Phys. Rev. A 71, 032317 (2005).

[12] C. F. Ockeloen-Korppi et al., Nature (London) 556, 478 (2018).

[13] S. Gröblacher, K. Hammerer, M. Vanner, and M. Aspelmeyer, Nature (London) 460, 724 (2009).

[14] M. Aspelmeyer, T. J. Kippenberg, and F. Marquardt, Rev. Mod. Phys. 86, 1391 (2014).

[15] C. Genes, D. Vitali, P. Tombesi, S. Gigan, and M. Aspelmeyer, Phys. Rev. A 77, 033804 (2008).

[16] E. Verhagen, S. Deleglise, S. Weis, A. Schliesser, and T. J. Kippenberg, Nature (London) 482, 63 (2012).

[17] T. A. Palomaki, J. D. Teufel, R. W. Simmonds, and K. W. Lehnert, Science 342, 710 (2013).

[18] D. Vitali, S. Gigan, A. Ferreira, H.R. Bohm, P. Tombesi, A. Guerreiro, V. Vedral, A. Zeilinger, and M. Aspelmeyer, Phys. Rev. Lett. 98, 030405 (2007).

[19] B. Julsgaad, A. Kozhekin, and E. S. Polzik, Nature (London) 413, 400 (2001).

[20] A. J. Berkley et al., Science 300, 1548 (2003).

[21] D. Lachance-Quirion et al., Sci. Adv. 3, e1603150 (2017).

[22] X. Zhang, C.-L. Zou, L. Jiang, and H. X. Tang, Sci. Adv. 2, e1501286 (2016).

[23] D. Zhang et al., npj Quantum Inf. 1, 15014 (2015).

[24] D. Lachance-Quirion, Y. Tabuchi, A. Gloppe, K. Usami, and Y. Nakamura, Appl. Phys. Express 12, 070101 (2019).

[25] Y. Tabuchi, S. Ishino, T. Ishikawa, R. Yamazaki, K. Usami, and Y. Nakamura, Phys. Rev. Lett. 113, 083603 (2014).
[26] Ö. O. Soykal and M. E. Flatte, Phys. Rev. Lett. 104, 077202 (2010).

[27] X. Zhang, C.-L. Zou, L. Jiang, and H. X. Tang, Phys. Rev. Lett. 113, 156401 (2014).

[28] J. Bourhill, N. Kostylev, M. Goryachev, D. L. Creedon, and M. E. Tobar, Phys. Rev. B 93, 144420 (2016).

[29] Y. Tabuchi et al., Science 349, 405 (2015).

[30] M. Harder, Y. Yang, B. M. Yao, C. H. Yu, J. W. Rao, Y. S. Gui, R. L. Stamps, and C. M. Hu, Phys. Rev. Lett. 121, 137203 (2018).

[31] B. Yao et al., Nat. Commun. 8, 1437 (2017).

[32] X. Zhang et al., Nat. Commun. 6, 8914 (2015).

[33] J. Li, S.-Y. Zhu, and G. S. Agarwal, Phys. Rev. Lett. 121, 203601 (2018).

[34] J. Li, S.-Y. Zhu, and G. S. Agarwal, Phys. Rev. A 99, 021801(R) (2019).

[35] J. Li and S.-Y. Zhu, New J. Phys. 21, 085001 (2019).

[36] Y. P. Wang, G. Q. Zhang, D. Zhang, T. F. Li, C. M. Hu, and J. Q. You, Phys. Rev. Lett. 120, 057202 (2018).

[37] L. Bai et al., Phys. Rev. Lett. 118, 217201 (2017).

[38] P. Hyde, B. M. Yao, Y. S. Gui, G.-Q. Zhang, J. Q. You, and C.-M. Hu, Phys. Rev. B 98, 174423 (2018).

[39] M. Sarovar, A. Ishizaki, G. R. Fleming, and K. Birgitta Whaley, Nat. Phys. 6, 462 (2010).

[40] Z. D. Zhang and J. Wang, Sci. Rep. 6, 37629 (2016).

[41] D. M. Coles et al., Nat. Mater. 13, 712 (2014).

[42] Z. D. Zhang, P. Saurabh, K. E. Dorfman, A. Debnath, and S. Mukamel, J. Chem. Phys. 148, 074302 (2018).

[43] M. Kowalewski, K. Bennett, and S. Mumakel, J. Phys. Chem. Lett. 7, 2050 (2016).

[44] C. Kittel, Phys. Rev. 73, 155 (1948).

[45] There is also an exception as in Ref. [23], where the cavity design can achieve the simultaneous excitation of the Kittel and magnetostatic modes. Nevertheless, it is worth noting that the magnetostatic modes may undergo an incoherent intraband relaxation process which results in a dramatically larger dephasing than the Kittel mode. The MS can be thereby ignored when working within the timescale of $\sim 1 \mu \mathrm{s}$.

[46] O. Madelung and T. C. Taylor, Introduction to Solid-State Theory (Springer-Verlag, Berlin, 1978).

[47] The magnetic dipole-dipole interaction between two YIG bulks is neglected, because such an interaction obeys the 
scaling of $(2 \pi r / \lambda)^{-3}$ with respect to distance. In recent experiments, the wavelength is about $30 \mathrm{~mm}$ and hence we can safely drop the dipolar coupling between YIGs when $r \gtrsim 1 \mathrm{~mm}$.

[48] E. M. Lifshitz and L. P. Pitaevskii, Statistical Physics: Theory of the Condensate State, Part 2, revised ed. (ButterworthHeinemann, Oxford, 1980).
[49] A. L. Fetter and J. D. Walecka, Quantum Theory of ManyParticle Systems (Dover Publications, Mineola, 2003).

[50] G. Vidal and R. F. Werner, Phys. Rev. A 65, 032314 (2002).

[51] R. Simon, Phys. Rev. Lett. 84, 2726 (2000).

[52] G. Adesso and F. Illuminati, New J. Phys. 8, 15 (2006).

[53] G. Adesso and F. Illuminati, J. Phys. A: Math. Theor. 40, 7821 (2007). 